\title{
RECONSTRUCTING DISCONTINUITIES USING COMPLEX GEOMETRICAL OPTICS SOLUTIONS*
}

\author{
GUNTHER UHLMANN ${ }^{\dagger}$ AND JENN-NAN WANG ${ }^{\ddagger}$
}

\begin{abstract}
In this paper we provide a framework for constructing general complex geometrical optics solutions for several systems of two variables that can be reduced to a system with the Laplacian as the leading order term. We apply these special solutions to the problem of reconstructing inclusions inside a domain filled with known conductivity from local boundary measurements. Computational results demonstrate the versatility of these solutions to determine electrical inclusions.
\end{abstract}

Key words. geometrical optics, inclusions, numerical algorithm

AMS subject classifications. 35R30, 65N21

DOI. $10.1137 / 060676350$

1. Introduction. Inverse boundary value problems are a class of inverse problems where one attempts to determine the internal parameters of body by making measurements only at the surface of the body. A prototypical example that has received a lot of attention is electrical impedance tomography (EIT). In this inverse method one would like to determine the conductivity distribution inside a body by making voltage and current measurements at the boundary.

There are many applications of EIT ranging from early breast cancer detection [32] to geophysical sensing for underground objects; see [18], [24], [25], [27]. The article [28] and the ones reviewed in [29] assume that the measurements are made on the whole boundary. However, it is often possible to make the measurements only on part of the boundary; this is the partial data problem. This is the case for the applications in breast cancer detection and geophysical sensing mentioned above.

The boundary information is encoded into the Dirichlet-to-Neumann map associated with the conductivity equation. More precisely, let $\Omega$ be an open bounded domain with smooth boundary in $\mathbb{R}^{d}$ with $d=2$ or 3 . Assume that $\gamma(x)>0$ in $\Omega$ possesses a suitable regularity. The conductivity equation is described by the following elliptic equation:

$$
\nabla \cdot(\gamma(x) \nabla u)=0 \text { in } \Omega .
$$

For an appropriate function $f$ defined on $\partial \Omega$, there exists a unique solution $u(x)$ to the boundary value problem for (1.1) with Dirichlet condition $\left.u\right|_{\partial \Omega}=f$. Thus, one can define a map $\Lambda_{\gamma}$ sending the Dirichlet data to the Neumann data by

$$
\Lambda_{\gamma}(f)=\left.\gamma \frac{\partial u}{\partial \nu}\right|_{\partial \Omega} .
$$

\footnotetext{
* Received by the editors November 30, 2006; accepted for publication (in revised form) October 16, 2007; published electronically February 29, 2008.

http://www.siam.org/journals/siap/68-4/67635.html

${ }^{\dagger}$ Department of Mathematics, University of Washington, Box 354305, Seattle, WA 98195-4350 (gunther@math.washington.edu). This author's research was partially supported by NSF and a Walker Family Endowed Professorship.

${ }^{\ddagger}$ Department of Mathematics, Taida Institute for Mathematical Sciences, and NCTS (Taipei), National Taiwan University, Taipei 106, Taiwan (jnwang@math.ntu.edu.tw). This author's research was supported in part by the National Science Council of Taiwan (NSC 95-2115-M-002-003).
}

1026 
The map $\Lambda_{\gamma}$ is the Dirichlet-to-Neumann map associated with the conductivity equation (1.1). It is worth mentioning that even though (1.1) is linear, the map $\Lambda_{\gamma}$ depends nonlinearly on $\gamma$. The famous Calderón problem is to determine $\gamma$ from the knowledge of $\Lambda_{\gamma}$.

In [3], Calderón studied this inverse problem by linearizing the fully nonlinear problem around a constant conductivity function. To attack this linearized problem, Calderón introduced harmonic functions of the form $e^{x \cdot \rho}$ with $\rho \in \mathbb{C}^{n}$ and $\rho \cdot \rho=0$, which is the genesis of complex geometrical optics (CGO) solutions since the phase function $x \cdot \rho$ is complex-valued. Inspired by Calderón's approach, Sylvester and Uhlmann [28] solved the uniqueness question of Calderón's problem for smooth conductivities by constructing CGO solutions for (1.1). Since the conductivity equation (1.1) is closely related to the Schrödinger equation (see (2.2)), it suffices to construct CGO solutions for the Schrödinger which are of the form $u(x)=e^{x \cdot \rho}(1+r(x, \rho))$, where $r$ is decaying in $|\rho|$. To motivate the name of the solution, we write

$$
u(x)=e^{i h^{-1} x \cdot\left(\omega_{1}+i \omega_{2}\right)}(1+h \tilde{r}),
$$

where $h=|\rho|^{-1}, i\left(\omega_{1}+i \omega_{2}\right)=|\rho|^{-1}(\operatorname{Re} \rho+i \operatorname{Im} \rho)$, and $\tilde{r}=h^{-1} r=|\rho| r$. The form (1.2) is analogous to the geometrical optics solution for the wave propagation equation in which the phase function is real-valued. Here the phase function in (1.2) is complex-valued. Nevertheless, it is linear. CGO solutions have been used in EIT and have been instrumental in solving several inverse problems. We will not review these developments in detail here; see [30] and [29] for references; other reviews in EIT are [1], [2], and [4].

Recently, new CGO solutions that are useful for the partial data problem were constructed in [20] for the conductivity equation and zeroth order perturbations of the Laplacian. The real parts of the phase of these solutions are limiting Carleman weights. They have been generalized to first order perturbation of the Laplacian for scalar equations or systems in [5], [9], [26], and [31]. Constructions of CGO solutions for the conductivity equation and zeroth order perturbations of the Laplacian using hyperbolic geometry can be found in [16], [17]; these have been applied to determine electrical inclusions in [10].

In two dimensions, when the underlying equation has the Laplacian as the leading part, due to the rich conformal structure, we have more freedom of choosing the complex phases for the CGO solutions. In particular any harmonic function is a limiting Carleman weight and can be the real part of a CGO solution. The aim of the paper is to provide a framework for constructing these solutions for several systems of two variables that can be reduced to a system with the Laplacian as the leading term. We apply these special solutions to the problem of reconstructing inclusions inside a domain filled with known conductivity from local boundary measurements. We also provide numerical results to demonstrate the applicability and flexibility of these special solutions.

From now on, we consider the case $d=2$, i.e., the $\mathbb{R}^{2}$ plane. Let $n \in \mathbb{N}$ and denote $U(x)=\left(u_{1}\left(x_{1}, x_{2}\right), \ldots, u_{n}\left(x_{1}, x_{2}\right)\right)^{\top}$. We consider the following system of equations:

$$
P U:=\Delta_{x} U+A_{1}(x) \partial_{x_{1}} U+A_{2}(x) \partial_{x_{2}} U+Q(x) U=0 \quad \text { in } \Omega,
$$

where $\Delta_{x}=\partial_{x_{1}}^{2}+\partial_{x_{2}}^{2}$ and $A_{1}, A_{2}, Q$ are $n \times n$ matrices whose regularities will be specified later. The system (1.3) contains all scalar or two-dimensional physical systems that can be reduced to a system with the Laplacian as the leading part. Those 
systems include the conductivity equation, the magnetic Schrödinger equation, the two-dimensional isotropic elasticity system, the two-dimensional Stokes system, etc. In this paper we first study CGO solutions with special phase functions for (1.3).

In the papers [20], [5], [9], [10], [17], [26], and [31], the real parts of the phase functions are radial functions. These can be used to probe the region with spherical fronts, the so-called complex spherical waves. Even though these solutions are better suited for the local data problem than the usual CGO solutions with linear phase functions, they are still quite restrictive. Fortunately, in the two-dimensional case, we have many more choices of phase functions. For example, let $\varphi(x)$ be a harmonic function with nonvanishing gradient in $\Omega$; then $\varphi+i \psi$ can be the phase function of the CGO solutions when $\psi$ is a harmonic conjugate of $\varphi$. In other words, $\rho(x):=$ $\varphi(x)+i \psi(x)$ is holomorphic in $\Omega$. Our method in this paper is developed based on this idea.

Using the CGO solutions, we can consider the problem of finding embedded inclusions in a known medium. This is the object identification problem. The method developed here shares the same spirit as Ikehata's enclosure method [11], [12]. For the two-dimensional problem, we would like to mention a very interesting result by Ikehata in [14], where he introduced the Mittag-Leffler function in the object identification problem. This has the property that its modulus grows exponentially in some cone and decays to zero algebraically outside the same cone. Using the Mittag-Leffler function and shrinking the opening angle of the cone, one can reconstruct precisely the shapes of some embedded objects such as star-shaped objects. The numerical implementation of the Mittag-Leffler functions was carried out by Ikehata and Siltanen in [15]. The main restriction of the method using the Mittag-Leffler function is that it can be applied only to scalar equations with a homogeneous background. That is, they probe the region with harmonic functions. The novelty of our method is its flexibility in treating scalar equations, or even two-dimensional systems, with an inhomogeneous background. Furthermore, for the object identification problem in such general systems, using our special CGO solutions, we are able to reconstruct the precise information of some embedded objects including star-shaped regions by boundary measurements. This identification result is similar to that in [14] and [15], where only the Laplace equation is treated. So, in theory, our reconstruction method with these CGO solutions is in greater generality. In this paper, we are developing the foundational work to treat the case of an inhomogeneous background and also to deal with the case of systems. Moreover, we give numerical evidence that the method works in the homogeneous case.

Before going further, we also would like to compare our method with that in [10]. As we have pointed out above, the real parts of the phase functions of CGO solutions in [10] are radially symmetric. So their probing fronts are circles or spheres. Moreover, the construction of CGO solutions in [10] is based on the hyperbolic geometry. It has not been developed for studying more general equations or systems. The advantage of our method lies in the freedom of choosing the phase functions of CGO solutions. One useful example is to take $\rho(x)$ as a polynomial. By increasing the degree of the polynomial, we can narrow our probing fronts. Consequently, we are able to determine more information in the object identification problem in the two-dimensional case than [10] does. On the other hand, since the real parts of the phase functions in our CGO solutions are not necessarily radially symmetric, we can create different probing fronts by simply rotating the phase functions. Like [10], we can also localize the measurements in an arbitrarily small region on the boundary. Here the local data means that the Dirichlet condition is nonzero only on a small part of the boundary. 
On the same region, we measure the Neumann condition. In theory, the nonzero part of the Dirichlet data can be taken as small as we wish.

Our construction of CGO solutions with more general phases is rather elementary. The main idea is to transform CGO solutions with linear phases by suitable conformal mappings. The construction of CGO solutions with linear phases for (1.3) was first given by Nakamura and Uhlmann in [21], [22], where they introduced the intertwining technique in handling the first order terms (also see [7] for similar results). Here we shall use Carleman's technique to construct CGO solutions with linear phases for (1.3).

This paper is organized a follows. In section 2, we give concrete examples of (1.3). In section 3, we review of the construction of CGO solutions with linear phases for (1.3). CGO solutions with more general phases will be discussed in section 4. For an application of CGO solutions with general phases, we consider the problem of reconstructing inclusions embedded into a domain with known conductivity by boundary measurements. Numerical experiments of our method are presented in section 6 .

\section{Physical examples of (1.3).}

2.1. Conductivity equation. Our first example is the well-known conductivity equation already given in the previous section. Let $\gamma(x) \in C^{2}(\bar{\Omega})$ and $\gamma(x)>0$ for all $x \in \bar{\Omega}$. We consider the equation

$$
\nabla \cdot(\gamma \nabla u)=0 \text { in } \Omega .
$$

Introducing the new variable $v=\gamma^{1 / 2} u,(2.1)$ is equivalent to

$$
(\Delta+q) v=0 \text { in } \Omega
$$

with $q=-\Delta \gamma^{1 / 2} / \gamma^{1 / 2} \in L^{\infty}(\Omega)$. Equation (2.2) is a Schrödinger-type equation. We can also consider a more general Schrödinger-type equation with a convection term:

$$
(\Delta+a(x) \cdot \nabla+q) v=0 \quad \text { in } \Omega,
$$

where $a=\left(a_{1}, a_{2}\right)$.

2.2. Isotropic elasticity. The domain $\Omega$ is now modeled as an inhomogeneous, isotropic, elastic medium characterized by the Lamé parameters $\lambda(x)$ and $\mu(x)$. Assume that $\lambda(x) \in C^{2}(\bar{\Omega}), \mu(x) \in C^{4}(\bar{\Omega})$, and the following inequalities hold:

$$
\mu(x)>0 \quad \text { and } \quad \lambda(x)+2 \mu(x)>0 \quad \forall x \in \bar{\Omega} \quad \text { (strong ellipticity) }
$$

We consider the static isotropic elasticity system without sources

$$
\nabla \cdot(\lambda(\nabla \cdot u) I+2 \mu S(\nabla u))=0 \quad \text { in } \quad \Omega .
$$

Here and below, $S(A)=\left(A+A^{T}\right) / 2$ denotes the symmetric part of the matrix $A \in$ $\mathbb{C}^{2 \times 2}$. Equivalently, if we denote $\sigma(u)=\lambda(\nabla \cdot u) I+2 \mu S(\nabla u)$ the stress tensor, then (2.5) becomes

$$
\nabla \cdot \sigma=0 \text { in } \Omega .
$$

On the other hand, since the Lamé parameters are differentiable, we can also write (2.5) in the nondivergence form

$$
\mu \Delta u+(\lambda+\mu) \nabla(\nabla \cdot u)+\nabla \lambda \nabla \cdot u+2 S(\nabla u) \nabla \mu=0 \quad \text { in } \quad \Omega .
$$


We will use the reduced system derived by Ikehata [13]. This reduction was also mentioned in [29]. Let $\left(\begin{array}{c}w \\ g\end{array}\right)$ satisfy

$$
\Delta\left(\begin{array}{c}
w \\
g
\end{array}\right)+A(x)\left(\begin{array}{c}
\nabla g \\
\nabla \cdot w
\end{array}\right)+Q(x)\left(\begin{array}{c}
w \\
g
\end{array}\right)=0
$$

where

$$
A(x)=\left(\begin{array}{cc}
2 \mu^{-1 / 2}\left(-\nabla^{2}+\Delta\right) \mu^{-1} & -\nabla \log \mu \\
0 & \frac{\lambda+\mu}{\lambda+2 \mu} \mu^{1 / 2}
\end{array}\right)
$$

and

$$
Q(x)=\left(\begin{array}{cc}
-\mu^{-1 / 2}\left(2 \nabla^{2}+\Delta\right) \mu^{1 / 2} & 2 \mu^{-5 / 2}\left(\nabla^{2}-\Delta\right) \mu \nabla \mu \\
-\frac{\lambda-\mu}{\lambda+2 \mu}\left(\nabla \mu^{1 / 2}\right)^{T} & -\mu \Delta \mu^{-1}
\end{array}\right) .
$$

Here $\nabla^{2} f$ is the Hessian of the scalar function $f$. Then

$$
u:=\mu^{-1 / 2} w+\mu^{-1} \nabla g-g \nabla \mu^{-1}
$$

satisfies (2.6). A similar form was also used in [7] for studying the inverse boundary value problem for the isotropic elasticity system.

2.3. Stokes system. Let $\mu(x) \in C^{4}(\bar{\Omega})$ and $\mu(x)>0$ for all $x \in \bar{\Omega}$. Here $\mu$ is called the viscosity function. Suppose that $u=\left(u_{1}, u_{2}\right)$ and $p$ satisfy the Stokes system

$$
\left\{\begin{array}{l}
\nabla \cdot(\mu S(\nabla u))-\nabla p=0 \quad \text { in } \quad \Omega \\
\nabla \cdot u=0 \text { in } \Omega .
\end{array}\right.
$$

Here $u$ and $p$ represent the velocity field and the pressure, respectively. Motivated by the isotropic elasticity, we set $u=\mu^{-1 / 2} w+\mu^{-1} \nabla g-\left(\nabla \mu^{-1}\right) g$ and

$$
p=\nabla \mu^{1 / 2} \cdot w+\mu^{1 / 2} \nabla \cdot w+2 \Delta g=\nabla \cdot\left(\mu^{1 / 2} w\right)+2 \Delta g
$$

then $(u, p)$ is a solution of $(2.8)$, provided $\left(\begin{array}{c}w \\ g\end{array}\right)$ satisfies

$$
\Delta\left(\begin{array}{c}
w \\
g
\end{array}\right)+A(x)\left(\begin{array}{c}
\nabla g \\
\nabla \cdot w
\end{array}\right)+Q(x)\left(\begin{array}{c}
w \\
g
\end{array}\right)=0
$$

with

$$
A(x)=\left(\begin{array}{cc}
-2 \mu^{1 / 2} \nabla^{2} \mu^{-1} & -\mu^{-1} \nabla \mu \\
0 & \mu^{1 / 2}
\end{array}\right)
$$

and

$$
Q=\left(\begin{array}{cc}
-2 \mu^{-1 / 2} \nabla^{2} \mu^{1 / 2}-\mu^{-1 / 2} \Delta \mu^{1 / 2} & -4 \nabla^{2} \mu^{-1} \nabla \mu^{1 / 2}-2 \mu^{1 / 2} \nabla \cdot\left(\nabla \mu^{-1}\right) \\
\mu\left(\nabla \mu^{-1 / 2}\right)^{T} & -\mu \Delta \mu^{-1}
\end{array}\right) .
$$

Copyright $@$ by SIAM. Unauthorized reproduction of this article is prohibited. 
3. CGO solutions with linear phases. In this section we review the method of constructing CGO solutions with linear phases using Carleman estimates. We consider a slightly different system here. Let $\tilde{\Omega}$ be an open bounded domain in $\mathbb{R}^{2}$. Let $V(y)=$ $V\left(y_{1}, y_{2}\right)$ satisfy

$$
\Delta_{y} V+\tilde{A}_{1} \partial_{y_{1}} V+\tilde{A}_{2} \partial_{y_{2}} V+\tilde{Q} V=0 \quad \text { in } \quad \tilde{\Omega} .
$$

Assume that $\tilde{A}_{1}, \tilde{A}_{2} \in C^{2}(\overline{\tilde{\Omega}})$ and $\tilde{Q} \in L^{\infty}(\tilde{\Omega})$. Given $\omega \in \mathbb{R}^{2}$ with $|\omega|=1$, we look for $V(y)$ of (3.1) having the form

$$
V(y)=e^{y \cdot\left(\omega+i \omega^{\perp}\right) / h}(\tilde{L}+\tilde{R}),
$$

where $\tilde{L}$ is independent of $h$ and $\tilde{R}$ satisfies

$$
\left\|\partial^{\alpha} \tilde{R}\right\|_{L^{2}(\tilde{\Omega})} \leq C h^{1-\alpha} \quad \forall|\alpha| \leq 2 .
$$

To construct $V$ having the form (3.2), (3.3), we follow the approach in [9] and [31], which are based on [5] and [20]. Note that the real part of the phase function $y \cdot \omega$ is a limiting Carleman estimate. So if we define the semiclassical operator

$$
P_{h}=h^{2} \Delta+h \tilde{A}_{1}\left(h \partial_{y_{1}}\right)+h \tilde{A}_{2}\left(h \partial_{y_{2}}\right)+h^{2} \tilde{Q},
$$

then we can derive, by combining a Carleman estimate and the Hahn-Banach theorem, the following.

TheOrem 3.1 (see [9], [31]). For $h$ sufficiently small, for any $F \in L^{2}(\tilde{\Omega})$, there exists $W \in H_{h}^{2}(\tilde{\Omega})$ such that

$$
e^{-y \cdot \omega / h} P_{h}\left(e^{y \cdot \omega / h} W\right)=F
$$

and $h\|W\|_{H_{h}^{2}(\tilde{\Omega})} \leq C\|F\|_{L^{2}(\tilde{\Omega})}$, where $\|W\|_{H_{h}^{2}(\tilde{\Omega})}^{2}=\sum_{|\alpha| \leq 2}\left\|(h \partial)^{\alpha} W\right\|_{L^{2}(\tilde{\Omega})}^{2}$ is the semiclassical $H^{2}$ norm.

This theorem will be needed below. Finding $V$ of the form (3.2) is equivalent to solving

$$
e^{-y \cdot\left(\omega+i \omega^{\perp}\right) / h} P_{h}\left(e^{y \cdot\left(\omega+i \omega^{\perp}\right) / h}(\tilde{L}+\tilde{R})\right)=0 \quad \text { in } \quad \tilde{\Omega} .
$$

We can compute that

$$
e^{-y \cdot\left(\omega+i \omega^{\perp}\right) / h} P_{h} e^{y \cdot\left(\omega+i \omega^{\perp}\right) / h}=h T_{\omega}+P_{h},
$$

where $T_{\omega}=2\left(\omega+i \omega^{\perp}\right) \cdot \nabla+\left(\omega+i \omega^{\perp}\right) \cdot\left(\tilde{A}_{1}, \tilde{A}_{2}\right)$. Hence we want to find $\tilde{L}$, independent of $h$, so that

$$
T_{\omega} \tilde{L}=0 \quad \text { in } \quad \tilde{\Omega} .
$$

Equation (3.4) is a system of Cauchy-Riemann type. In fact, introducing the new variable $z=\left(z_{1}, z_{2}\right)=\left(\omega+i \omega^{\perp}\right) \cdot y$ and setting $\tilde{A}(\omega, z)=\left(\omega+i \omega^{\perp}\right) \cdot\left(\tilde{A}_{1}, \tilde{A}_{2}\right),(3.4)$ becomes

$$
\left(4 \partial_{\bar{z}}+\tilde{A}\right) \tilde{L}=0
$$

where $\partial_{\bar{z}}=\left(\partial_{z_{1}}+i \partial_{z_{2}}\right) / 2$. The existence of nontrivial $\tilde{L}$ can be found in, for example, [6], [8], and [23]. Having found $\tilde{L}, \tilde{R}$ is required to satisfy

$$
e^{-y \cdot \omega / h} P_{h}\left(e^{y \cdot\left(\omega+i \omega^{\perp}\right) / h} \tilde{R}\right)=-e^{i y \cdot \omega^{\perp} / h} P_{h} \tilde{L} .
$$

Copyright $@$ by SIAM. Unauthorized reproduction of this article is prohibited. 
Note that $\left\|e^{i y \cdot \omega^{\perp} / h} P_{h} \tilde{L}\right\|_{L^{2}(\tilde{\Omega})}=O\left(h^{2}\right)$. Thus Theorem 3.1 implies that

$$
\left\|e^{i y \cdot \omega^{\perp} / h} \tilde{R}\right\|_{H_{h}^{2}(\tilde{\Omega})} \leq C h,
$$

which leads to

$$
\left\|\partial^{\alpha} \tilde{R}\right\|_{L^{2}(\tilde{\Omega})} \leq C h^{1-|\alpha|} \text { for }|\alpha| \leq 2 .
$$

REMARK 3.2. The leading term $\tilde{L}$ of the CGO solution (3.2) is obtained by solving (3.5). It is possible to solve (3.5) by an iteration scheme, which is numerically feasible. Theorem 3.1 is a general theorem to guarantee the existence of the remainder term $\tilde{R}$ in (3.2). It may be a nontrivial task to actually find $\tilde{R}$ for general systems. However, since $\tilde{R}$ is $O(h)$ for small $h$, it could be omitted in numerical computations.

4. CGO solutions with general phases. In this section we will construct CGO solutions with more general phases for (1.3) from CGO solutions with linear phases given in the previous section. Without loss of generality, we choose $\omega=(1,0)$ and $\omega^{\perp}=(0,1)$, i.e., $y \cdot\left(\omega+i \omega^{\perp}\right)=y_{1}+i y_{2}$. Denote $y=y_{1}+i y_{2}$ and $x=x_{1}+i x_{2}$. Let $\Omega_{0}$ be an open subdomain of $\Omega$. Suppose that $A_{1}, A_{2} \in C^{2}\left(\bar{\Omega}_{0}\right)$ and $Q \in L^{\infty}\left(\Omega_{0}\right)$. Let $y=\rho(x)=y_{1}\left(x_{1}, x_{2}\right)+i y_{2}\left(x_{1}, x_{2}\right)$ be a conformal map in $\Omega_{0}$, i.e., $\rho^{\prime}(x) \neq 0$ for all $x \in \Omega_{0}$. Define $U(x)=V(y(x))$ and $\tilde{\Omega}=\rho\left(\Omega_{0}\right)$. By straightforward computations, we have

$$
\left(\begin{array}{c}
\partial_{x_{1}} \\
\partial_{x_{2}}
\end{array}\right) U=\left.J(x)\left(\begin{array}{c}
\partial_{y_{1}} \\
\partial_{y_{2}}
\end{array}\right) V\right|_{y=\rho(x)} \quad \text { and } \quad \Delta_{x} U=\Delta_{y} V\left|\rho^{\prime}(x)\right|^{2}
$$

where

$$
J(x)=\left(\begin{array}{ll}
\partial_{x_{1}} y_{1} & \partial_{x_{1}} y_{2} \\
\partial_{x_{2}} y_{1} & \partial_{x_{2}} y_{2}
\end{array}\right)
$$

Suppose that $\rho^{-1}$ exists on $\tilde{\Omega}$. Let $\hat{A}_{1}(y)=\left(A_{1} \partial_{x_{1}} y_{1}+A_{2} \partial_{x_{2}} y_{1}\right) \circ \rho^{-1}(y), \hat{A}_{2}(y)=$ $\left(A_{1} \partial_{x_{1}} y_{2}+A_{2} \partial_{x_{2}} y_{2}\right) \circ \rho^{-1}(y)$, and $\hat{Q}(y)=\left(Q \circ \rho^{-1}\right)(y)$ and $g(y)=\left|\left(\rho^{\prime} \circ \rho^{-1}\right)(y)\right|^{2}$. Now if we choose $V(y)$ satisfying

$$
\Delta_{y} V+g(y)^{-1} \hat{A}_{1}(y) \partial_{y_{1}} V+g(y)^{-1} \hat{A}_{2}(y) \partial_{y_{2}} V+g(y)^{-1} \hat{Q} V=0 \quad \text { in } \tilde{\Omega},
$$

then $U(x)$ satisfies (1.3) in $\Omega_{0}$. According to the construction given previously, let $V(y)$ be a solution of (4.1) having the form

$$
V(y)=e^{\left(y_{1}+i y_{2}\right) / h}(\tilde{L}+\tilde{R}),
$$

where

$$
\left\|\partial^{\alpha} \tilde{R}\right\|_{L^{2}(\tilde{\Omega})} \leq C h^{1-\alpha} \quad \forall|\alpha| \leq 2 .
$$

Denote $y_{1}\left(x_{1}, x_{2}\right)=\varphi\left(x_{1}, x_{2}\right)$ and $y_{2}\left(x_{1}, x_{2}\right)=\psi\left(x_{1}, x_{2}\right)$. We then obtain CGO solutions for (1.3) in $\Omega_{0}$ :

$$
U(x)=e^{(\varphi+i \psi) / h}(L+R)
$$

with $L=\tilde{L} \circ \rho, R=\tilde{R} \circ \rho$, and

$$
\left\|\partial^{\alpha} R\right\|_{L^{2}\left(\Omega_{0}\right)} \leq C h^{1-\alpha} \quad \forall|\alpha| \leq 2 .
$$

Copyright $@$ by SIAM. Unauthorized reproduction of this article is prohibited. 
Due to the conformality of $\rho, \varphi$ and $\psi$ are harmonic functions in $\Omega_{0}$. Conversely, given any $\varphi$ harmonic in $\Omega_{0}$ with $\nabla \varphi \neq 0$ in $\Omega_{0}$, we can find a harmonic conjugate $\psi$ of $\varphi$ in $\Omega_{0}$ so that $\rho=\varphi+i \psi$ is conformal in $\Omega_{0}$. The freedom of choosing $\varphi$ plays a key role in our reconstruction method for the object identification problem. Actually, we will mainly focus on the level curves of $\varphi$. We give some concrete examples here.

Pick a point $x_{0} \notin \bar{\Omega}$. It is no restriction to assume that $x_{0}=0$. We now consider $\varphi_{N}=\operatorname{Re}\left(c_{N} x^{N}\right)$ for $N \geq 2$, where $c_{N} \in \mathbb{C}$ with $\left|c_{N}\right|=1$. In the polar coordinates, $\varphi_{N}(r, \theta)=r^{N} \cos N\left(\theta-\theta_{N}\right)$ for some $\theta_{N}$ determined by $c_{N}$. We observe that $\varphi_{N}>0$ in some open cone $\Gamma_{N}$ with an opening angle $\pi / N$. The freedom of choosing $\theta_{N}$ (or, equivalently, $c_{N}$ ) allows us to "sweep" the domain $\Omega$ by $\Gamma_{N}$ without moving the point $x_{0}$. This is quite useful in practice. Now assume that $\Gamma_{N} \cap \Omega \neq \emptyset$. The complex function $\rho_{N}(x)=c_{N} x^{N}=\varphi_{N}+i \psi_{N}$ is clearly conformal in $\Omega$, where $\psi_{N}=\operatorname{Im}\left(c_{N} x^{N}\right)$. In order to apply to the inverse problem, we want to shrink the opening angle of $\Gamma_{N}$ by taking $N \rightarrow \infty$. However, there are two serious problems in doing so. On one hand, $\varphi_{N}$ is periodic in the angular variable, which means that it is positive in some other cones with the same opening angle which also intersect $\Omega$ when $N$ is large. Some level curves of $\varphi_{N}$ for different $N$ 's are shown in Figure 4.1. This property of $\varphi_{N}$ prohibits us from using corresponding CGO solutions with large $N$ to the object identification problem. On the other hand, the complex function $\rho_{N}(x)$ fails to be injective in the whole domain $\Omega$ when $N$ is large. To overcome those difficulties and construct useful CGO solutions in the whole domain $\Omega$, we shall carry out the construction described above in a suitable $\Omega_{0}$ and extend the constructed solutions to $\Omega$ by cut-off functions.
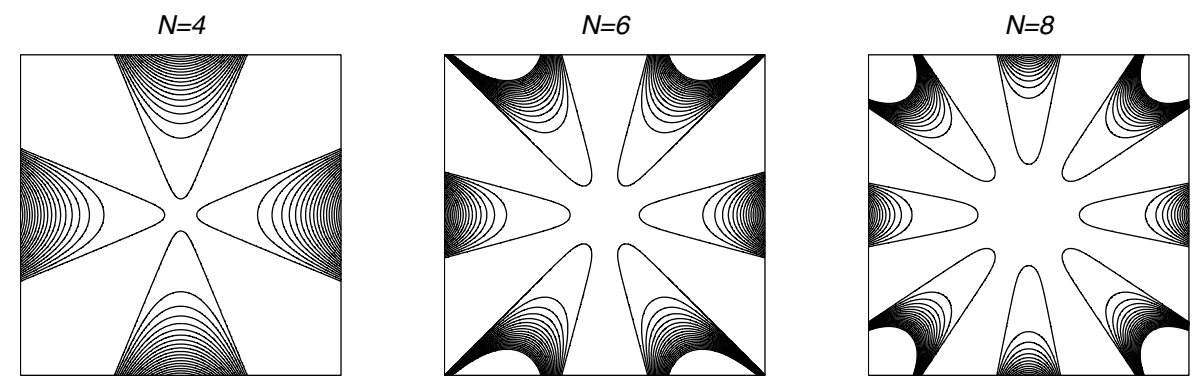

FIG. 4.1. Some level curves of $\phi_{N}$.

We now set

$$
\Omega_{0}:=\Gamma_{N} \cap \Omega .
$$

Then $\rho_{N}$ is conformal in $\Omega_{0}$ and is bijective from $\Omega_{0}$ onto $\rho_{N}\left(\Omega_{0}\right)$. Therefore, we can find CGO solutions for (1.3) in $\Omega_{0}$,

$$
U_{N, h}(x)=e^{\left(\varphi_{N}+i \psi_{N}\right) / h}(L+R),
$$

and the estimate (4.2) holds. So far we have constructed only special solutions for (1.3) in some particular subdomain of $\Omega$. To get solutions in the whole domain $\Omega$, we use a cut-off technique. For $s>0$, let $\ell_{s}=\left\{x \in \Gamma_{N}: \varphi_{N}=s^{-1}\right\}$. This is the level curve of $\varphi_{N}$ in $\Gamma_{N}$. Let $0<t<t_{0}$ such that

$$
\left(\underset{s \in(0, t)}{\cup} \ell_{s}\right) \cap \Omega \neq \emptyset
$$


and choose a small $\varepsilon>0$. Define a cut-off function $\phi_{N, t}(x) \in C^{\infty}\left(\mathbb{R}^{2}\right)$ so that $\phi_{N, t}(x)=1$ for $x \in \overline{\left(\cup_{s \in(0, t+\varepsilon / 2)} \ell_{s}\right) \cap \Omega}$ and is zero for $x \in \bar{\Omega} \backslash\left(\cup_{s \in(0, t+\varepsilon)} \ell_{s}\right)$. We now define

$$
U_{N, t, h}(x)=\phi_{N, t} e^{-t^{-1} / h} U_{N}=\phi_{N, t} e^{\left(\varphi_{N}-t^{-1}+i \psi_{N}\right) / h}(L+R)
$$

for $x \in\left(\cup_{s \in(0, t+\varepsilon)} \ell_{s}\right) \cap \Omega$. So $U_{N, t, h}$ can be regarded as a function in $\Omega$ which is zero outside of $\Omega_{0}$. We now take $f_{N, t, h}=\left.U_{N, t, h}\right|_{\partial \Omega}$. We remark that $f_{N, t, h}$ can be used as the boundary data in the inverse problem. An obvious reason for using $f_{N, t, h}$ is that they are local.

Now we define a function $W:=W_{N, t, h}$ satisfying

$$
\left\{\begin{array}{l}
\Delta W+A_{1}(x) \partial_{x_{1}} W+A_{2}(x) \partial_{x_{2}} W+Q(x) W=0 \text { in } \Omega, \\
W=f_{N, t, h} \text { on } \partial \Omega .
\end{array}\right.
$$

We would like to compare $W_{N, t, h}$ with $U_{N, t, h}$. It turns out they differ only by an exponentially small term under some minor condition. This property plays an essential role in our method for the inverse problem.

Lemma 4.1. Assume that the boundary value problem

$$
\left\{\begin{array}{l}
P U=0 \quad \text { in } \quad \Omega \\
U=0 \quad \text { on } \quad \partial \Omega
\end{array}\right.
$$

has only a trivial solution. Then there exist $C>0$ and $\varepsilon^{\prime}>0$ such that

$$
\left\|W_{N, t, h}-U_{N, t, h}\right\|_{H^{2}(\Omega)} \leq C e^{-\varepsilon^{\prime} / h}
$$

for $h \ll 1$.

Proof. By setting $G:=W_{N, t, h}-U_{N, t, h}$, we get that

$$
\begin{aligned}
P G & =P\left(W_{N, t, h}-U_{N, t, h}\right) \\
& =-\phi_{N, t} e^{-t^{-1} / h} P U_{N}+\left[\phi_{N, t}, P\right] e^{-t^{-1} / h} U_{N} \\
& =\left[\phi_{N, t}, P\right] e^{-t^{-1} / h} U_{N} \\
& =\left[\phi_{N, t}, P\right] e^{\left(\varphi_{N}-t^{-1}+i \psi_{N}\right) / h}(L+R)
\end{aligned}
$$

since $P U_{N}=0$ in $\left(\cup_{s \in\left(0, t_{0}\right)} \ell_{s}\right) \cap \Omega$. Now we observe that $\left[\phi_{N, t}, P\right]$, the commutator of $\phi_{N, t}$ and $P$, is a first order differential operator with coefficients supported in

$$
\overline{\left(\underset{s \in(t+\varepsilon / 2, t+\varepsilon)}{\cup} \ell_{s}\right) \cap \Omega} \text {. }
$$

So we have that

$$
\left\|\left[\phi_{N, t}, P\right] e^{\left(\varphi_{N}-t^{-1}+i \psi_{N}\right) / h}(L+R)\right\|_{L^{2}(\Omega)} \leq C^{\prime} e^{-\varepsilon^{\prime} / h}
$$

for some $C^{\prime}>0$ and $\varepsilon^{\prime}>0$. Note that $G=0$ on $\partial \Omega$. Combining the regularity theorem, the triviality of (4.4), and (4.6) yields (4.5).

Even though the solutions $W_{N, t, h}$ of (1.3) are not exactly in the form of complex geometrical optics, with the help of Lemma 4.1, they are exponentially close to $U_{N, t, h}$. Now we describe how to construct special solutions for some concrete systems given

Copyright $@$ by SIAM. Unauthorized reproduction of this article is prohibited. 
in section 2 from $W_{N, t, h}$. For the conductivity equation (2.1), (1.3) is reduced to (2.2). For (2.2), we denote the corresponding $U_{N, t, h}=u_{N, t, h}$ and

$$
u_{N, t, h}=\phi_{N, t} e^{\left(\varphi_{N}-t^{-1}+i \psi_{N}\right) / h}(1+r),
$$

where $r$ satisfies (4.2). With $u_{N, t, h}$, we can solve for $w_{N, t, h}$ satisfying

$$
\left\{\begin{array}{l}
(\Delta+q) w=0 \quad \text { in } \Omega, \\
w=u_{N, t, h} \text { on } \partial \Omega .
\end{array}\right.
$$

The problem (4.7) has a unique solution since the boundary value problem for the corresponding conductivity equation has a unique solution. So Lemma 4.1 implies that

$$
\left\|w_{N, t, h}-u_{N, t, h}\right\|_{H^{1}(\Omega)} \leq C e^{-\varepsilon^{\prime} / h} .
$$

Returning to the conductivity equation, we see that $\gamma^{-1 / 2} w_{N, t, h}$ are solutions of (2.1).

For the isotropic elasticity and the Stokes system, we have that $n=3$ and (1.3) become, respectively, (2.7) and (2.10). We discuss only the isotropic elasticity here. The Stokes system can be treated similarly. Assume that the homogeneous boundary value problem (4.4) associated with (2.7) has only the trivial solution. Thus Lemma 4.1 yields

$$
\left\|W_{N, t, h}-U_{N, t, h}\right\|_{H^{2}(\Omega)} \leq C e^{-\varepsilon^{\prime} / h} .
$$

We now express $U_{N, t, h}=\left(\begin{array}{c}v_{N, t, h} \\ b_{N, t, h}\end{array}\right)$ and $W_{N, t, h}=\left(\begin{array}{c}w_{N, t, h} \\ g_{N, t, h}\end{array}\right)$, where $v_{N, t, h}, w_{N, t, h}$ are two-dimensional vectors and $b_{N, t, h}, g_{N, t, h}$ are scalars. Hence, we obtain that

$$
u_{N, t, h}=\mu^{-1 / 2} w_{N, t, h}+\mu^{-1} \nabla g_{N, t, h}-g_{N, t, h} \nabla \mu^{-1}
$$

are solutions of (2.6) or (2.5) and $u_{N, t, h}$ satisfies

$$
\left\|u_{N, t, h}-\left(\mu^{-1 / 2} v_{N, t, h}+\mu^{-1} \nabla b_{N, t, h}-b_{N, t, h} \nabla \mu^{-1}\right)\right\|_{H^{1}(\Omega)} \leq C e^{-\varepsilon^{\prime} / h} .
$$

5. Inverse problems. In this section we demonstrate how to use CGO solutions constructed previously in the object identification problem. To simplify our presentation, we will discuss only the case of identifying inclusions inside of the domain $\Omega$ filled with known conductivity. This inverse problem has been extensively studied both theoretically and numerically. We refer the reader to [10] for related references. Using our method, we can also treat the object identification problem for other systems. We shall report the results elsewhere.

Let $D$ be an open bounded domain with $C^{1}$ boundary such that $\bar{D} \subset \Omega$ and $\Omega \backslash \bar{D}$ is connected. Assume $\gamma(x) \in C^{2}(\bar{\Omega})$ with $\gamma(x)>0$ for all $x \in \bar{\Omega}$. The conductivity $\tilde{\gamma}(x)$ is a perturbation of $\gamma$ described by $\tilde{\gamma}(x)=\gamma+\chi_{D} \gamma_{1}$, where $\chi_{D}$ is the characteristic function of $D$ and $\gamma_{1} \in C(\bar{D})$. We suppose that

$$
\gamma_{1} \geq 0 \quad \text { in } \quad D \text { and } \gamma_{1}>0 \text { on } \partial D \text {. }
$$

Then we have $\tilde{\gamma}(x) \geq c>0$ almost everywhere in $\Omega$. Let $v$ be the solution of

$$
\left\{\begin{array}{l}
\nabla \cdot(\tilde{\gamma} \nabla v)=0 \text { in } \Omega \\
v=f \text { on } \partial \Omega .
\end{array}\right.
$$

Copyright (C) by SIAM. Unauthorized reproduction of this article is prohibited. 
The meaning of the solution to (5.2) is understood in the following way. Define

$$
[w]_{\partial D}=\operatorname{tr}^{+} w-\operatorname{tr}^{-} w
$$

the jump of the function across $\partial D$, where $\operatorname{tr}^{+}$and $\operatorname{tr}^{-}$denote, respectively, the trace of $w$ on $\partial D$ from inside and outside of $D$. For $f \in H^{3 / 2}(\partial \Omega)$, we define

$$
\mathcal{V}_{f}=\left\{w \in H^{2}(D) \oplus H^{2}(\Omega \backslash \bar{D}):\left.w\right|_{\partial \Omega}=f,[w]_{\partial D}=0,\left[\tilde{\gamma} \frac{\partial w}{\partial \nu}\right]_{\partial D}=0\right\} .
$$

We say that $v$ is the solution of (5.2) if $v \in \mathcal{V}_{f}$ and $\nabla \cdot(\tilde{\gamma} v)=0$ in $D$ and $\Omega \backslash \bar{D}$. The Dirichlet-to-Neumann map is given as

$$
\Lambda_{D}:\left.f \rightarrow \tilde{\gamma} \frac{\partial v}{\partial \nu}\right|_{\partial \Omega}
$$

where $\nu$ is the unit outer normal of $\partial \Omega$. The inverse problem is to determine the inclusion $D$ from $\Lambda_{D}$. Here we are interested in the reconstruction question.

Since our method shares the same spirit as Ikehata's enclosure method [11], [12], we will briefly describe Ikehata's ideas to motivate our method. Here we take $\gamma \equiv 1$, i.e., $\tilde{\gamma}=1+\chi_{D} \gamma_{1}$. Denote

$$
f_{\omega}(x, \tau, t)=\exp \left\{\tau(x \cdot \omega-t)+i \tau x \cdot \omega^{\perp}\right\}
$$

and

$$
I_{\omega}(\tau, t)=\left\langle\left(\Lambda_{D}-\Lambda_{0}\right) f_{\omega}(\cdot, \tau, t), \overline{f_{\omega}(\cdot, \tau, t)}\right\rangle,
$$

where $\Lambda_{0}$ is the Dirichlet-to-Neumann map associated with $\Delta u=0$ in $\Omega$. Let us define

$$
h_{D}(\omega)=\sup _{x \in D} x \cdot \omega .
$$

Then the following formulas hold:

$$
\left\{t \in \mathbb{R}: \lim _{\tau \rightarrow 0} I_{\omega}(\tau, t)=0\right\}=\left(h_{D}(\omega), \infty\right)
$$

and

$$
\lim _{\tau \rightarrow \infty} \frac{\log \left|\tau_{\omega}(\tau, t)\right|}{2 \tau}=h_{D}(\omega)-t \quad \forall t \in \mathbb{R}
$$

(see [11], [12]).

To describe our method, we begin with the following integral inequalities given in [19] (also see [10] for a proof).

Lemma 5.1. Assume that (5.6) holds. Let $f \in H^{3 / 2}(\partial \Omega)$ and $u$ be the unique solution of

$$
\left\{\begin{array}{l}
\nabla \cdot(\gamma \nabla u)=0 \quad \text { in } \quad \Omega \\
u=f \text { on } \partial \Omega .
\end{array}\right.
$$

Define $\Lambda_{0}:\left.f \rightarrow \gamma \frac{\partial u}{\partial \nu}\right|_{\partial \Omega}$. Then we have

$$
\int_{\partial \Omega}\left(\Lambda_{D}-\Lambda_{0}\right) \bar{f} \cdot f d s \leq \int_{D} \gamma_{1}|\nabla u|^{2} d x
$$

Copyright $@$ by SIAM. Unauthorized reproduction of this article is prohibited. 
and

$$
\int_{\partial \Omega}\left(\Lambda_{D}-\Lambda_{0}\right) \bar{f} \cdot f d s \geq \int_{D} \frac{\gamma_{1} \gamma}{\gamma+\gamma_{1}}|\nabla u|^{2} d x .
$$

It follows from (5.1) that for any $p \in \partial D$, there exists an $\epsilon>0$ such that

$$
\gamma_{1} \geq \epsilon \quad \forall x \in D \cap B_{\epsilon}(p) .
$$

Let $x_{0} \notin \bar{\Omega}$ and define the open cone $\Gamma_{N}$ with $\Gamma_{N} \cap \Omega \neq \emptyset$ in terms of $\varphi_{N}=$ $\operatorname{Re}\left(c_{N}\left(x-x_{0}\right)^{N}\right)\left(\rho_{N}=c_{N}\left(x-x_{0}\right)^{N}\right)$ as in Figure 4.1. Likewise, we denote the level curve $\ell_{s}=\left\{x \in \Gamma_{N}: \varphi_{N}=s^{-1}\right\}$ for $s>0$. For $\varepsilon>0$ and $t>0$, we take

$$
f=f_{N, t, h}=\left.\gamma^{-1 / 2} w_{N, t, h}\right|_{\partial \Omega}=\left.\gamma^{-1 / 2} u_{N, t, h}\right|_{\partial \Omega}
$$

where $w_{N, t, h}$ and $u_{N, t, h}$ are constructed previously. Note that $\gamma^{-1 / 2} w_{N, t, h}$ is the solution of (5.3). It should be noted that the Dirichlet condition $f$ is localized in $\Gamma_{N} \cap \partial \Omega$ and supp $(f)$ becomes narrower as $N$ gets bigger. This property is very useful in actual applications.

To construct the inclusion $D$, we rely on the quantity

$$
E(N, t, h):=\int_{\partial \Omega}\left(\Lambda_{D}-\Lambda_{0}\right) \bar{f}_{N, t, h} \cdot f_{N, t, h} d s .
$$

Clearly, this quantity is completely determined by the boundary data. From (5.1) and (5.5) we see that

$$
E(N, t, h) \geq \int_{D} \frac{\gamma_{1} \gamma}{\gamma+\gamma_{1}}\left|\nabla\left(\gamma^{-1 / 2} w_{N, t, h}\right)\right|^{2} d x \geq 0
$$

for all $N, t, h$. We now prove the following important behavior of $E(N, t, h)$.

TheOREM 5.2. Let $t>0$ and $\mathcal{L}_{t}=\left\{x \in \Gamma_{N}: \varphi_{N} \geq t^{-1}\right\}$. Then we have the following:

(i) if $\mathcal{L}_{t} \cap \bar{D}=\emptyset$, then there exist $C_{1}>0, \varepsilon_{1}>0$, and $h_{1}>0$ such that $E(N, t, h) \leq C_{1} e^{-\varepsilon_{1} / h}$ for all $h \leq h_{1}$;

(ii) if $\mathcal{L}_{t} \cap D \neq \emptyset$, then there exist $C_{2}>0, \varepsilon_{2}>0$, and $h_{2}>0$ such that $E(N, t, h) \geq C_{2} e^{\varepsilon_{2} / h}$ for all $h \leq h_{2}$.

Proof. To prove (i), we use the inequality (5.4) to obtain

$$
E(N, t, h) \leq \int_{D} \gamma_{1}\left|\nabla\left(\gamma^{-1 / 2} w_{N, t, h}\right)\right|^{2} d x \leq C\left\|w_{N, t, h}\right\|_{H^{1}(D)}^{2}
$$

With the help of (4.8), we can replace $w_{N, t, h}$ in (5.9) by $u_{N, t, h}$ with an error $O\left(e^{-\varepsilon^{\prime} / h}\right)$. Since $\mathcal{L}_{t} \cap \bar{D}=\emptyset$, we have $\varphi_{N}-t^{-1}<0$ for all $x \in \bar{D} \cap \Gamma_{N}$. Also, note that $u_{N, t, h} \equiv 0$ in $\Omega \backslash \Gamma_{N}$. Therefore, by the form of $u_{N, t, h}$ we immediately derive that

$$
E(N, t, h) \leq C e^{-\varepsilon_{1} / h}
$$

for $h \leq h_{1}$.

To establish (ii), in view of $\mathcal{L}_{t} \cap D \neq \emptyset$, there exist $z \in \partial D$ and $\epsilon>0$ such that the jump condition (5.6) holds and

$$
\varphi_{N}-t^{-1} \geq \epsilon \quad \forall B_{\epsilon}(z) \cap D
$$

Copyright (C) by SIAM. Unauthorized reproduction of this article is prohibited. 
From (5.5) we get

$$
\begin{aligned}
E(N, t, h) & \geq \int_{D} \frac{\gamma_{1} \gamma}{\gamma+\gamma_{1}}\left|\nabla\left(\gamma^{-1 / 2} w_{N, t, h}\right)\right|^{2} d x \\
& \geq C \epsilon \int_{D \cap B_{\epsilon}(z)}\left(\left|\nabla w_{N, t, h}\right|^{2}+\left|w_{N, t, h}\right|^{2}\right) d x \\
& \geq C^{\prime} \int_{D \cap B_{\epsilon}(z)}\left(\left|\nabla u_{N, t, h}\right|^{2}+\left|u_{N, t, h}\right|^{2}\right) d x-C^{\prime \prime} e^{-\varepsilon^{\prime} / h} .
\end{aligned}
$$

Substituting the form of $u_{N, t, h}$ with the estimate (5.10) into (5.11) implies the statement of (ii).

THEOREM 5.3. With the same notation as in Theorem 5.2, if $\ell_{t} \cap \partial D \neq \emptyset$ and $\mathcal{L}_{t} \cap D=\emptyset$, then

$$
\lim \inf _{h \rightarrow 0} E(N, t, h)>0 .
$$

Recall that $\ell_{t}=\left\{x \in \Gamma_{N}: \varphi_{N}=t^{-1}\right\}$.

Proof. In view of (5.6), we pick a sufficiently small $\epsilon>0$ such that (5.6) is satisfied in $B_{\epsilon}(p) \cap D$ and $B_{\epsilon}(p) \cap D \subset\left(\cup_{s \in(t, t+\varepsilon / 2)} \ell_{s}\right) \cap D$. So the cut-off function $\phi_{N, t}=1$ on $B_{\epsilon}(p) \cap D$. We now introduce a new coordinate system $\Psi(x)=\left(y_{1}(x), y_{2}(x)\right)$ near $p$ with $y_{2}(x)=\varphi_{N}-t^{-1}$ such that $\ell_{t}$ becomes $y_{2}=0$ near $p$ and $\tilde{D}_{\epsilon}:=\Psi\left(B_{\epsilon}(p) \cap D\right)$ lies in $\left\{y_{2}<0\right\}$. We can choose a small cone $C_{p}$ in $\tilde{D}_{\epsilon}$ with vertex $p$ and the length of the axis being $\delta$. Denote $J(y)$ the Jacobian of $\Psi^{-1}(y)$. Therefore, using (5.11) we can estimate

$$
\begin{aligned}
& E(N, t, h) \\
\geq & C^{\prime} \int_{D \cap B_{\epsilon}(p)}\left(\left|\nabla u_{N, t, h}\right|^{2}+\left|u_{N, t, h}\right|^{2}\right) d x-C^{\prime \prime} e^{-\varepsilon^{\prime} / h} \\
\geq & C^{\prime} \int_{D \cap B_{\epsilon}(p)}\left(\left|\nabla\left(e^{\left(\varphi_{N}-t^{-1}+i \psi_{N}\right) / h}(1+r)\right)\right|^{2}+\left|e^{\left(\varphi_{N}-t^{-1}+i \psi_{N}\right) / h}(1+r)\right|^{2}\right) d x \\
& -C^{\prime \prime} e^{-\varepsilon^{\prime} / h} \\
\geq & \frac{\tilde{C}}{h^{2}} \int_{C_{p}} e^{2 y_{2} / h}|J| d y_{1} d y_{2}-C^{\prime \prime} e^{-\varepsilon^{\prime} / h} \\
\geq & \frac{\tilde{C}^{\prime}}{h^{2}} \int_{-\delta}^{0} e^{2 y_{2} / h} y_{2} d y_{2}-C^{\prime \prime} e^{-\varepsilon^{\prime} / h} \\
> & 0 \quad \text { as } h \rightarrow 0 .
\end{aligned}
$$

In view of Theorems 5.2 and 5.3, we are able to reconstruct some part of $\partial D$ by looking into the asymptotic behavior of $E(N, t, h)$ for various $t$ 's. More precisely, let

$$
t_{D, N}:=\sup \left\{t \in(0, \infty): \lim _{h \rightarrow 0} E(N, h, t)=0\right\}
$$

then if $t_{D, N}=\infty$, we have $\Gamma_{N} \cap D=\emptyset$. On the other hand, if $t_{D, N}<\infty$, then there exists a $p_{D, N} \in \ell_{t_{D, N}} \cap \partial D$.

By taking $N$ arbitrarily large (the opening angle of $\Gamma_{N}$ becomes arbitrarily small), we can reconstruct even more information of $\partial D$. A point $p$ on $\partial D$ is said to be detectable if there exists a half-line $l$ starting from $p$ such that $l$ does not intersect $\partial D$ except at $p$. For example, if $D$ is star-shaped, every point of $\partial D$ is detectable.

Copyright $@$ by SIAM. Unauthorized reproduction of this article is prohibited. 
COROLlary 5.4. Every detectable point of $\partial D$ can be reconstructed from $\Lambda_{D}$.

Proof. Let $p$ be a detectable point and $l$ be the corresponding half-line. We can choose $l$ which is not tangent to $\partial D$ at $p$ since if the chosen half-line, say $l^{\prime}$, is tangent to $\partial D$ at $p$, we can always choose a desired $l$ by perturbing $l^{\prime}$ a little bit. Assume that $z_{0} \in l$ and $z_{0} \neq p$. Let $L$ be the straight line containing $l$. Pick a point $x_{0} \in L$ with $\frac{x_{0}-p}{\left|x_{0}-p\right|}=-\frac{z_{0}-p}{\left|z_{0}-p\right|}$ and $x_{0} \notin \bar{\Omega}$. Let $\Gamma_{N}$ be the cone with axis $L$ and vertex $x_{0}$ whose opening angle is $\pi / N$. For any $N \in \mathbb{N}$, we construct $w_{N, t, h}, u_{N, t, h}$, and $f_{N, t, h}$ as above. So we can determine $E(N, t, h)$ from the measurement $\Lambda_{D} f_{N, t, h}$. Applying Theorems 5.2 and 5.3 , we can determine $t_{D, N}$ so that $\ell_{t_{D, N}} \cap \partial D \neq \emptyset$. Then there exists $p_{N} \in \Gamma_{N}$, and $\ell_{t_{D, N}} \cap \partial D=p_{N}$. By taking $N \rightarrow \infty$, we can see that $p_{N} \rightarrow p$.

To end this section, we give an algorithm of our reconstruction method based on Theorem 5.2.

Step 1. Pick a point $x_{0} \notin \bar{\Omega}$ (but close to $\bar{\Omega}$ ). Given $N \in \mathbb{N}$, choose the cone $\Gamma_{N}$ which intersects $\Omega$. $\left[\Gamma_{N}\right.$ is defined in section 4$]$

Step 2. Start with $t>0$ such that $\ell_{t} \cap \Omega \neq \emptyset$. Construct $u_{N, t, h}$ and determine the Dirichlet data $f_{N, t, h}=\left.\gamma^{-1 / 2} u_{N, t, h}\right|_{\partial \Omega}$. [(5.7)]

Step 3. Compute $E(N, t, h)=\int_{\text {supp }\left(f_{N, t, h}\right)}\left(\Lambda_{D}-\Lambda_{0}\right) \bar{f}_{N, t, h} \cdot f_{N, t, h} d s .[(5.8)]$

Step 4. If $E(N, t, h)$ is arbitrarily small, then increase $t$ and repeat Steps 2 and 3; if $E(N, t, h)$ is arbitrarily large, then decrease $t$ and repeat Steps 2 and 3. [Theorem 5.2]

Step 5. Repeat Step 4 to get a good approximation of $\partial D$ in $\Gamma_{N}$. [Theorem 5.2]

Step 6. Move the cone $\Gamma_{N}$ around $x_{0}$ by taking a different $c_{N}$ in $\varphi_{N}=\operatorname{Re}\left(c_{N} x^{N}\right)$. Repeat Steps 2-5.

Step 7. Choose a larger $N$ and a new cone $\Gamma_{N}$. Repeat Steps 2-6.

Step 8. Pick a different $x_{0}$ and repeat Steps 1-7.

6. Numerical results. We demonstrate some numerical results of our method in this section. Assume that the domain $\Omega$ is given by

$$
\Omega=\left\{\left(x_{1}, x_{2}\right):-1<x_{1}<1,-1.01<x_{2}<-0.1\right\} .
$$

We shall use the Dirichlet data localized on $\left\{\left(x_{1},-1.01\right):-1<x<1\right\}$. To set up $\rho_{N}(x)$, we consider $N=4$; i.e., the phase function of the CGO solution is $\rho(x):=$ $\rho_{4}(x)$. In our numerical computations, we use two sweeping schemes. In the first scheme, we fix the reference point $x_{0}$ and rotate the "probing cone" (the cone with the vertex at $x_{0}$ and the opening angle $\pi / 4$ ). For the second one, we do not rotate the probing cone but move the reference points along the $x$-axis. More precisely, let the reference point $x_{0}=\left(x_{0,1}, 0\right)$ for $-1<x_{0,1}<1$. In our first scheme, we fix $x_{0}=(0,0)$ and rotate the probing cone determined by the shifted angle $\theta$; in the second scheme, we consider different $x_{0}$ 's and choose $\theta=0$. In other words, for both schemes, we have

$$
\rho\left(x, x_{0}, \theta\right):=c(\theta)\left(x_{1}-x_{0,1}+i x_{2}\right)^{4}=e^{-i 4 \theta}\left(x_{1}-x_{0,1}+i x_{2}\right)^{4} .
$$

Thus, the probing fronts are level curves of $\varphi\left(x, x_{0}, \theta\right):=\operatorname{Re}\left(\rho\left(x, x_{0}, \theta\right)\right)$. Figure 6.1 shows some probing fronts of $\varphi\left(x, x_{0}, \theta\right)$ with three different $\theta$ 's and three $x_{0}$ 's, respectively.

We take the background conductivity $\gamma=1$, and the conductivity inside the inclusion is 4 , i.e, $\gamma_{1}=3$. For numerical experiments, we ignore the cut-off function and take 

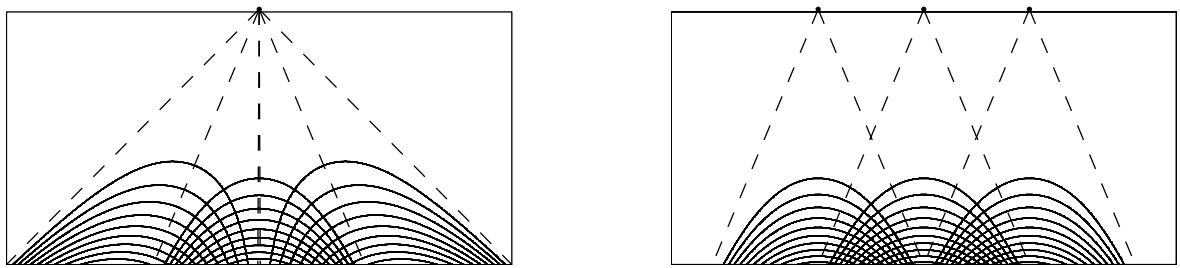

Fig. 6.1. Probing fronts of our numerical method. In the first column, we consider the probing cone in three different angles. In the second column, we move the probing cone by taking three reference points. In our numerical method, we use 10 different probing cones.

$$
\left.g_{x_{0}, h}\right|_{\partial \Omega}=\left\{\begin{array}{l}
e^{\rho\left(x, x_{0}, \theta\right) / h} \text { for }\left(x_{1}, x_{2}\right) \in \partial \Omega_{\mathrm{obs}}, \\
0, \quad \partial \Omega \backslash \partial \Omega_{\mathrm{obs}},
\end{array}\right.
$$

where $\partial \Omega_{\mathrm{obs}}$ is determined by $x_{0}$ and $\theta$. For example, for $x_{0}=(0,0)$ and $\theta=0$,

$$
\partial \Omega_{\mathrm{obs}}=\left\{\left(x_{1}, x_{2}\right):-1.01 \times \tan \left(\frac{\pi}{8}\right)<x_{1}<1.01 \times \tan \left(\frac{\pi}{8}\right), x_{2}=-1.01\right\} .
$$

Then for $t>0$ the required Dirichlet data is given by $f=f_{t, h, x_{0}}=e^{-t^{-1} / h} g_{x_{0}, h}$. To get the synthetic data $\Lambda_{0} f$ and $\Lambda_{D} f$, we need to solve the boundary value problems (5.2) and (5.3) with the Dirichlet condition $f$. To solve these forward problems, we use the PDE Toolbox with the finite element method in MATLAB 7.0. Since we need to collect data on the bottom boundary of $\Omega$, we refine the mesh there; see Figure 6.2.

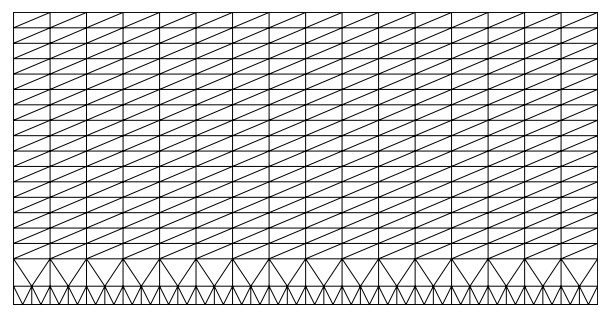

FIG. 6.2. Example of our finite element method meshes. The mesh has $2^{m}+1$ nodes on the top boundary and $2^{n}+1$ nodes on the lower boundary. This example is created with $m=4, n=6$. In solving our forward problems, we choose $m=6, n=12$.

To show the effect of noise to our method, we add appropriate noise to the synthetic data. We consider the form of noise given in [10]. To be precise, let $\eta:[-1,1] \mapsto$ $\mathbb{C}$ be a random function defined by

$$
\eta(s)=\sum_{k=-32}^{32}\left(a_{k}+i b_{k}\right) e^{i k s \pi / 2},
$$

where $a_{k}, b_{k} \sim \mathcal{N}(0,1)$ are normally distributed random numbers. The number 32 in $\eta$ is chosen to roughly model a collection of 32 electrodes on the bottom boundary of $\Omega$. Measurement noise is modeled by $\Lambda_{D} f$ by $\Lambda_{D} f+c \eta$ with

$$
c=\frac{A\left\|\Lambda_{D} f\right\|_{\infty}}{\|\eta\|_{\infty}}
$$

where $A>0$. 
Our strategy of reconstructing the inclusion is described as follows. We first design $M$ probing cones which are forms by taking either $M$ different vertex points or $M$ different rotating angles. Recall that each cone is congruent to the cone with its vertex at the origin and opening angle $\pi / 4$. We then take appropriate $h_{1}$ and $h_{2}$ with $h_{1}>h_{2}$ and choose a suitable number of probing fronts determined by $t_{j}$ for $j=1, \ldots, J$ with $t_{j}<t_{j+1}$. In each probing cone $\Gamma_{m}(m=1, \ldots, M)$ given above, we construct the Dirichlet data $f$ supported in the intersection of $\Gamma_{m}$ and the bottom boundary of $\partial \Omega$ for every $h_{k}$ and $t_{j}, k=1,2, j=1, \ldots, J$. We now evaluate $E_{j, k}:=E\left(N, t_{j}, h_{k}\right)$ and determine $t_{n}$ such that

$$
E_{n+1,2}>E_{n+1,1} .
$$

Then the region $R_{m}$ defined by

$$
R_{m}=\left\{x \in \Gamma_{m}: \varphi\left(x, x_{0}, \theta\right) \leq t_{n}^{-1}\right\}
$$

is the estimated largest region in $\Gamma_{m}$ which does not contain the inclusion. So the region $R:=\cup_{m=1}^{M} R_{m}$ is the estimated largest region with the absence of the inclusion with a given sweeping scheme. We would like to point out that condition (6.1) is our rule of thumb in determining whether the level curve $\varphi\left(x, x_{0}, \theta\right)=t^{-1}$ intersects the inclusion in our numerical experiments. It is not equivalent to Theorem 5.2 but is based on the reasoning that $E(N, t, h)$ is exponentially decaying when $\varphi\left(x, x_{0}, \theta\right)=t^{-1}$ stays away from the inclusion and exponentially growing when $\varphi\left(x, x_{0}, \theta\right)=t^{-1}$ intersects the inclusion. A similar idea was also used in [10].

Our numerical results for each sweeping scheme are shown in Figures 6.3 and 6.4. To save computational time, we show only numerical results obtained from probing
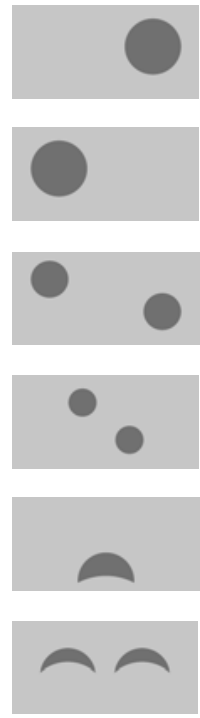
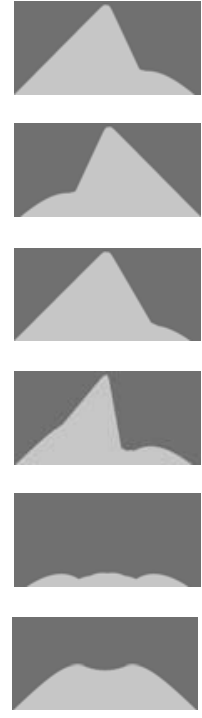
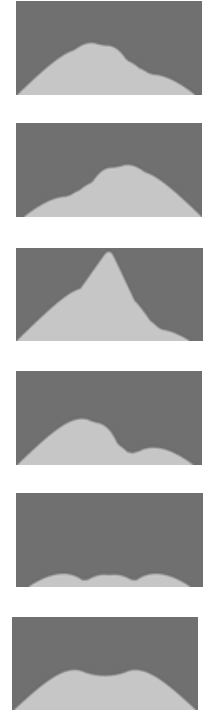
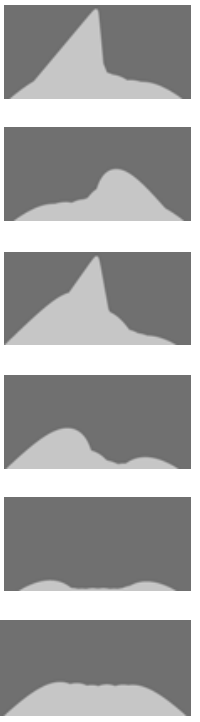

FIG. 6.3. Numerical results of the first sweeping scheme. All black regions have the conductivity 4, and all gray regions have conductivity 1 . So the gray regions represent the inclusion-free regions. The first column represents the actual location of inclusions. The second column is the theoretical reconstruction when we probe the region only from the bottom. The third column represents the numerical reconstruction from noiseless synthetic data. The fourth column is the numerical reconstruction from data with $0.01 \%$ noise. To see the effectiveness of our method, we can compare the images in the third column or in the fourth column with those in the second column. 

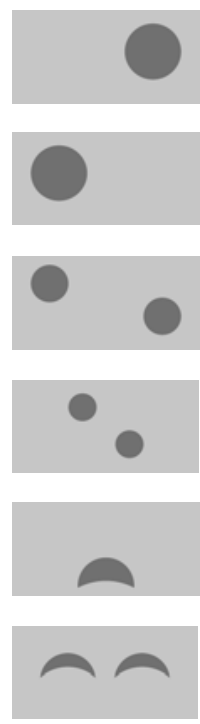
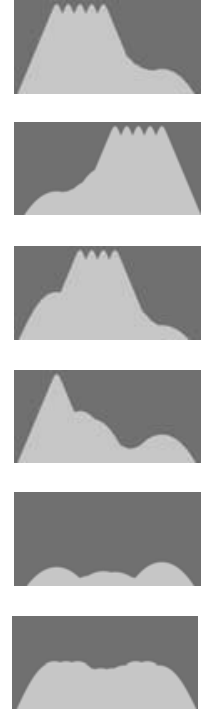
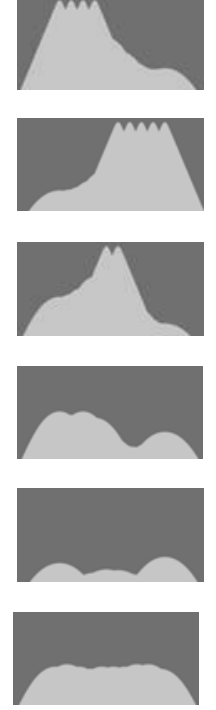
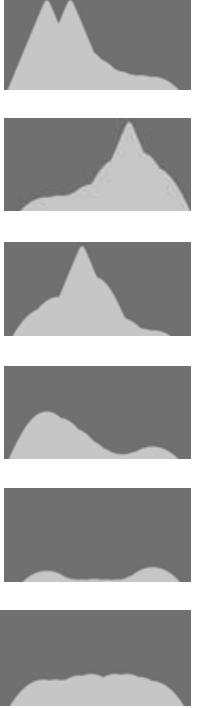

Fig. 6.4. Numerical results of the second sweeping scheme. All black regions have the conductivity 4, and all gray regions have conductivity 1 . So the gray regions represent the inclusion-free regions. The first column represents the actual location of inclusions. The second column is the theoretical reconstruction when we probe the region only from the bottom. The third column represents the numerical reconstruction from noiseless synthetic data. The fourth column is the numerical reconstruction from data with $0.01 \%$ noise. To see the effectiveness of our method, we can compare the images in the third column or in the fourth column with those in the second column.

the region from one side (the bottom part of the boundary). Therefore, the inclusionfree region (with gray color) is near the bottom of the boundary. Since our domain is a rectangle, we can expect to obtain similar results when we probe the region from other sides. We believe that these numerical results are sufficient to demonstrate the applicability of our method.

7. Conclusion. In this work we present a framework of constructing special complex geometrical optics solutions for several systems of two variables that can be reduced to a system with the Laplacian as the leading term. Here we choose complex polynomials as phase functions. Using these special solutions, we design a novel algorithm to identify embedded objects with boundary measurements. One distinctive feature of our method is that we can probe the region using cones with as small an opening angle as we wish. Theoretically, we are able to reconstruct the exact geometry of the embedded object whose boundary points are all detectable. One typical example is the star-shaped object.

In the numerical experiments, we consider the case of inclusion embedded into a domain with homogeneous conductivity. The numerical results show that our method detects the location of inclusion quite well and is stable under measurements with (small) noise. For computational reasons, we consider only $N=4$ and use two sweeping schemes separately. It is quite natural to consider higher $N$ 's and also combine two sweeping schemes into one. Of course, by doing so, we need to pay the price of increasing computational time.

Our method can be applied to classes of equations or even systems in two dimensions that can be reduced to the Laplacian on the top order part. Its flexibility and 
effectiveness gives us another technique that can potentially be used in real applications such as medical imaging or nondestructive evaluation.

Acknowledgments. The program codes used in this work are modified from those used in [10]. We would like to thank H. Isozaki, T. Ide, S. Nakata, and S. Siltanen for generously sharing the codes with us. In completing the numerical results of this paper, the second author benefited from conversations with Z. Li, R. LeVeque, G. Nakamura, K. M. Shyue, and C. T. Wu. We also thank the referees and the editor for carefully reading the manuscript and for many valuable suggestions.

\section{REFERENCES}

[1] L. BorceA, Electrical impedance tomography, Inverse Problems, 18 (2002), pp. R99-R136.

[2] L. BorceA, Addendum to: Electrical impedance tomography, Inverse Problems, 19 (2003), pp. 997-998.

[3] A. Calderón, On an inverse boundary value problem, in Seminar on Numerical Analysis and Its Applications to Continuum Physics, Sociedade Brasileira de Matemática, Río de Janeiro, Brazil, 1980, pp. 65-73.

[4] M. Cheney, D. Isaacson, and J. C. Newell, Electrical impedance tomography, SIAM Rev., 41 (1999), pp. 85-101.

[5] D. Dos Santos Ferreira, C. E. Kenig, J. Sjöstrand, And G. Uhlmann, Determining a magnetic Schrödinger operator from partial Cauchy data, Comm. Math. Phys., 271 (2007), pp. 467-488.

[6] G. Eskin, Global uniqueness in the inverse scattering problem for the Schrödinger operator with external Yang-Mills potentials, Comm. Math. Phys., 222 (2001), pp. 503-531.

[7] G. Eskin AND J. RALSTON, On the inverse boundary value problem for linear isotropic elasticity, Inverse Problems, 18 (2002), pp. 907-921.

[8] G. Eskin AND J. RAlston, On the inverse boundary value problem for linear isotropic elasticity and Cauchy-Riemann system, in Inverse Problems and Spectral Theory, Contemp. Math. 348, AMS, Providence, RI, 2004, pp. 53-69.

[9] H. Heck, G. Uhlmann, and J.-N. Wang, Reconstruction of obstacles immersed in an incompressible fluid, Inverse Probl. Imaging, 1 (2007), pp. 63-76.

[10] T. Ide, H. Isozaki, S. Nakata, S. Siltanen, And G. Uhlmann, Probing for electrical inclusions with complex spherical waves, Comm. Pure Appl. Math., 60 (2007), pp. 1415-1442.

[11] M. IKEHATA, Reconstruction of the support function for inclusion from boundary measurements, J. Inverse Ill-Posed Probl., 8 (2000), pp. 367-378.

[12] M. Ikehata AND S. Siltanen, Numerical method for finding the convex hull of an inclusion in conductivity from boundary measurements, Inverse Problems, 16 (2000), pp. 1043-1052.

[13] M. Ikehata, A Remark on an Inverse Boundary Value Problem Arising in Elasticity, preprint.

[14] M. IkehatA, Mittag-Leffler's function and extracting from Cauchy data, in Inverse Problems and Spectral Theory, Contemp. Math. 348, AMS, Providence, RI, 2004, pp. 41-52.

[15] M. IKehata And S. Siltanen, Electrical impedance tomography and Mittag-Leffler's function, Inverse Problems, 20 (2004), pp. 1325-1348.

[16] H. IsOzAKI, Inverse spectral problems on hyperbolic manifolds and their applications to inverse boundary value problems in Euclidean space, Amer. J. Math., 126 (2004), pp. 1261-1313.

[17] H. Isozaki And G. Uhlmann, Hyperbolic geometry and the local Dirichlet-to-Neumann map, Adv. Math., 188 (2004), pp. 294-314.

[18] J. Jordana, M. Gasulla, And R. Pallas-Areny, Electrical resistance tomography to detect leaks from buried pipes, Meas. Sci. Technol., 12 (2001), pp. 1061-1068.

[19] H. KAng, J. K. SEO, And D. Sheen, The inverse conductivity problem with one measurement: Stability and estimation of size, SIAM J. Math. Anal., 28 (1997), pp. 1389-1405.

[20] C. E. Kenig, J. Suöstrand, and G. Uhlmann, The Calderón problem with partial data, Ann. of Math. (2), 165 (2007), pp. 567-591.

[21] G. Nakamura And G. Uhlmann, Global uniqueness for an inverse boundary problem arising in elasticity, Invent. Math., 118 (1994), pp. 457-474.

[22] G. Nakamura and G. Uhlmann, Erratum: Global uniqueness for an inverse boundary value problem arising in elasticity, Invent. Math., 152 (2003), pp. 205-207.

[23] G. Nakamura and G. Uhlmann, Complex geometric optics solutions and pseudoanalytic matrices, in Ill-Posed and Inverse Problems, VSP, Zeist, The Netherlands, 2002, pp. 305-338. 
[24] A. Ramirez, W. Daily, D. LaBrecque, E. Owen, and D. Chesnut, Monitoring an underground steam injection process using electrical resistance tomography, Water Resour. Res., 29 (1993), pp. 73-87.

[25] A. Ramirez, W. Daily, A. Binley, D. LaBrecque, and D. Roelant, Detection of leaks in underground storage tanks using electrical resistance methods, J. Envir. Eng. Geophys., 1 (1996), pp. 189-203.

[26] M. Salo And J.-N. Wang, Complex spherical waves and inverse problems in unbounded domains, Inverse Problems, 22 (2006), pp. 2299-2309.

[27] L. Slater, A. M. Binley, W. Daily, And R. Johnson, Cross-hole electrical imaging of a controlled saline tracer injection, J. Appl. Geophys., 44 (2000), pp. 85-102.

[28] J. Sylvester and G. Uhlmann, A global uniqueness theorem for an inverse boundary value problem, Ann. of Math. (2), 125 (1987), pp. 153-169.

[29] G. Uhlmann, Developments in inverse problems since Calderón's foundational paper, in Harmonic Analysis and Partial Differential Equations (Essays in Honor of Alberto P. Calderón), The University of Chicago Press, Chicago, IL, 1999, pp. 295-345.

[30] G. Uhlmann, Commentary on Calderón's paper: On an inverse boundary value problem, in Selecta, Papers of Alberto P. Calderón, A. Bellow, C. E. Kenig, and P. Malliavin, eds.

[31] G. Uhlmann AND J.-N. WANG, Complex spherical waves for the elasticity system and probing of inclusions, SIAM J. Math. Anal., 38 (2007), pp. 1967-1980.

[32] Y. Zou AND Z. Guo, A review of electrical impedance techniques for breast cancer detection, Med. Eng. Phys., 25 (2003), pp. 79-90.

Copyright (C) by SIAM. Unauthorized reproduction of this article is prohibited. 